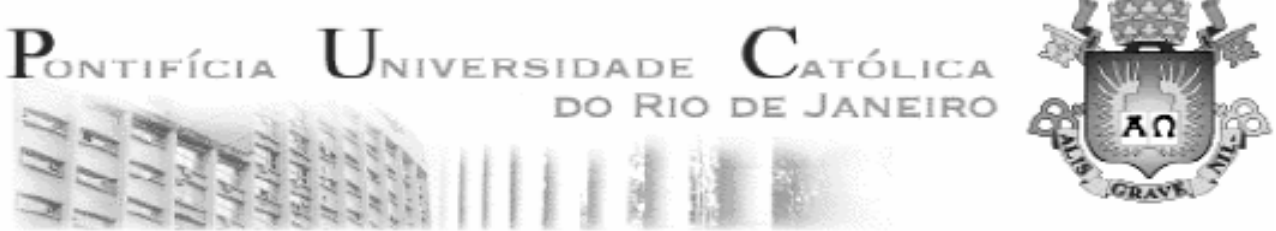

Tânia Oliveira Américo Pessôa

\title{
Avaliação da Influência da Mineralogia, Índice de Vazios e Teor de Umidade em Propriedades Térmicas de Solos
}

Dissertação de Mestrado

Dissertação apresentada como requisito parcial para obtenção do título de Mestre pelo Programa de PósGraduação em Engenharia Civil da PUC-Rio.

Orientadores: Tácio Mauro Pereira de Campos

Anna Paula Lougon Duarte

Franklin dos Santos Antunes

Rio de Janeiro, setembro de 2006. 


\section{Pontifícia Universidade Católica DO RIO DE JANEIRO}

Tânia Oliveira Américo Pessôa

\section{Avaliação da Influência da Mineralogia, Índice de Vazios e Teor de Umidade em Propriedades Térmicas de Solos}

Dissertação apresentada como requisito parcial para obtenção do título de Mestre pelo Programa de Pós-Graduação em Engenharia Civil da PUC-Rio. Aprovada pela Comissão Examinadora abaixo assinada.

Prof. Tácio Mauro Pereira de Campos

Presidente/Orientador

PUC-Rio

Anna Paula Lougon Duarte

Co-Orientadora

PUC-Rio

Prof. Franklin dos Santos Antunes Co-Orientador Departamento de Engenharia Civil - PUC-Rio

Prof. José T. Araruna Jr. Departamento de Engenharia Civil - PUC-Rio

Prof. Roberto F. de Azevedo UFV

Prof. José Eugênio Leal Coordenador Setorial do Centro Técnico Científico - PUC-Rio

Rio de Janeiro, 15 de setembro de 2006. 
Todos os direitos reservados. É proibida a reprodução total ou parcial do trabalho sem autorização da universidade, da autora e do orientador.

Tânia Oliveira Américo Pessôa

Engenheira Civil formada pela Universidade Federal de Sergipe - UFS em 2004.

Ficha Catalográfica

Pessôa, Tânia Oliveira Américo.

Avaliação da Influência da Mineralogia, Índice de Vazios e Teor de Umidade em Propriedades Térmicas de Solos / Tânia Oliveira Américo Pessôa; orientadores: Tácio Mauro Pereira de Campos, Anna Paula Lougon Duarte e Franklin dos Santos Antunes. Rio de Janeiro: PUC, Departamento de Engenharia Civil, 2006.

163f.: il; $30 \mathrm{~cm}$

Dissertação (mestrado) - Pontifícia Universidade Católica do Rio de Janeiro, Departamento de Engenharia Civil.

Inclui Referências Bibliográficas.

1. Engenharia Civil - Teses. 2. Propriedades térmicas. 3. Geotecnia ambiental. 4. Ensaios de laboratório. 5. Fluxo de calor em solos. I. de Campos Tácio Mauro Pereira. II. Duarte, Anna Paula Lougon. III. Antunes, Franklin dos Santos. IV. Pontifícia Universidade Católica do Rio de Janeiro. Departamento de Engenharia Civil. V. Título. 
Aos meus Pais, Luís e Salete, por todo amor e carinho, pelo exemplo de vida e pela educação que me deram.

Aos meus irmãos Patrícia, Susi e Fredy por serem meus amigos sempre. 


\section{Agradecimentos}

A Deus pela vida, pela saúde e pela família que tenho.

Aos Professores Tácio e Franklin pela orientação.

À Anna Paula por fazer parte desse trabalho e da minha vida de forma tão especial. Obrigada pela orientação, incentivo, paciência e amizade.

À CAPES, FAPERJ/CNPq (PRONEX-Rio) pelo apoio financeiro.

Aos professores do Departamento pelos conhecimentos transmitidos, em especial ao Prof. Franklin pelo ser humano que é.

À William, Sr. José, Amaury, Josué e Mônica Moncada pelo apoio no Laboratório de Geotecnia.

Aos funcionários do Departamento de Engenharia Civil, Ana Roxo, Rita, Cristiano e Lenilson.

Aos meus pais por serem meus espelhos e meus guias, por toda história de vida, exemplo de força, dignidade e generosidade. Aos meus irmãos por serem meus irmãos e meus amigos, e a Deus mais uma vez por fazer parte desta família.

À minha irmã Patrícia e ao seu esposo Ludimar por terem me acolhido em seu lar, por todo apoio, carinho e paciência de sempre. Não tenho como agradecer.

Aos meus professores da UFS pelos conhecimentos transmitidos, e em especial aos professores Demóstenes, Erinaldo, Sandra Dórea e Ângela Sales pelos profissionais que são e por terem despertado em mim o gosto pela Geotecnia e pela vida acadêmica.

Aos meus queridos e saudosos colegas da Graduação, Gustavo, Zenóbio, Manoel e Bruno pela amizade, companhia e ajuda de sempre. 
Aos meus colegas da Pós-Graduação, em especial às meninas, Elisangela, Taíse, Bernadete, Vivi e Carol pela amizade e apoio durante esses dois anos de curso.

Ao Homem da minha Vida, Júnior, por me trazer paz, alegria e muito amor. 


\section{Resumo}

Pessôa, Tânia Oliveira Américo; de Campos, Tácio Mauro Pereira; Duarte, Anna Paula Lougon; Antunes, Franklin dos Santos. Avaliação da Influência da Mineralogia, Índice de Vazios e Teor de Umidade em Propriedades Térmicas de Solos. Rio de Janeiro, 2006. 163p. Dissertação de Mestrado - Departamento de Engenharia Civil, Pontifícia Universidade Católica do Rio de Janeiro.

O conhecimento das propriedades térmicas dos solos é de grande importância em muitos projetos de engenharia e outras situações onde ocorre a transferência de calor nos solos, como em projetos de rodovias, de tubulações para petróleo, água, gás, cabos elétricos enterrados, aplicação de técnicas para remedição de solos contaminados, entre outros. A propagação de calor nos solos é governada pelas suas propriedades térmicas e essas propriedades devem ser determinadas experimentalmente. A presente Dissertação teve como objetivo principal a avaliação da influência da mineralogia, do índice de vazios e do teor de umidade em propriedades térmicas de solos. As principais propriedades térmicas dos solos são condutividade térmica, resistividade térmica, difusividade térmica e calor específico. Foram avaliados experimentalmente a condutividade, a difusividade, o calor específico dos solos e o calor específico dos sólidos. Trabalhou-se com misturas em diferentes proporções de materiais argilosos e arenosos. Os solos foram fabricados a partir de diferentes proporções de Caulim, Bentonita e Areia Quartzosa, formando-se nove solos diferentes. Os solos fabricados foram caracterizados física, química e mineralogicamente. Para a determinação da condutividade térmica e da difusividade térmica aplicou-se o método da sonda térmica de imersão em solos. O calor específico dos solos e o calor específico dos sólidos foram estimados através de equações propostas na literatura consultada. Os corpos de prova foram confeccionados por compactação estática e por vibração. Foi observado que os parâmetros térmicos são dependentes da umidade, do índice de vazios e da mineralogia dos solos de formas diferentes e, eventualmente, opostas, sendo a influência da umidade mais significativa. Foi proposta uma correlação para estimar o calor específico de areias quartzosas que depende apenas do teor de umidade gravimétrica.

\section{Palavras-chave}

Propriedades térmicas; geotecnia ambiental; ensaios de laboratório; fluxo de calor em solos. 


\section{Abstract}

Pessôa, Tânia Oliveira Américo; de Campos, Tácio Mauro Pereira (advisor); Duarte, Anna Paula Lougon (advisor); Antunes, Franklin dos Santos (advisor). Evaluation of Effects of Mineralogy, Voids Ratio and Moisture Content on Thermal Properties of Soils. Rio de Janeiro, 2006. 163p. MSc. Dissertation - Civil Eng. Dept., Pontifícia Universidade Católica do Rio de Janeiro.

The knowledge of thermal properties of soils is of great relevance in many engineering projects where the transference of heat in soils occurs, such as: projects of highways, oil, water and gas pipeways, electric cables embedded in the ground and application of techniques for remediation of contaminated soil. The propagation of heat in the ground is governed by its thermal properties and these properties should be determined by laboratory tests. This Dissertation has as main objective the evaluation of the influence of mineralogy, voids ratio and water content in thermal soil properties. The main thermal properties of soils are thermal conductivity, thermal resistivity, thermal difusivity and specific heat. Soil conductivity and difusivity were obtained experimentally, while the specific heat of soil and specific heat of solids were obtained through equations proposed in the currente literature. The soils used comprised mixtures in different ratios of clayey and sandy materials employing different ratios of commercially found Kaolin, Bentonite and Quartz pellets, forming nine different soils. The prepared soils were characterized in their physical, chemical and mineralogical aspects. For the determination of the thermal conductivity and the thermal diffusivity it was employed the methodology of insertion of a thermal needle in the soils. Both vibration and static compaction techniques were used in the preparation of the soil samples to be tested. It was observed that the thermal parameters are dependent on the water content, voids ratio and mineralogy of the soil in different and, eventually, opposite ways.

\section{Keywords}

Thermal properties; environmental geotechnical; laboratory tests ; heat flow in soil. 


\section{Sumário}

1 Introdução 21

1.1. Estrutura da Dissertação 22

2 Revisão Bibliográfica $\quad 24$

2.1. Propriedades Térmicas dos Solos $\quad 24$

2.1.1. Condutividade Térmica 27

2.1.2. Resistividade Térmica 32

2.1.3. Calor Específico e Capacidade de Aquecimento Volumétrico 33

2.1.4. Difusividade Térmica 37

2.2. Fatores que Influenciam as Propriedades Térmicas dos Solos 38

2.2.1. Estrutura e Porosidade dos Solos 39

2.2.2. Mudanças na Estrutura dos Solos 40

2.2.3. A Água nos Solos $\quad 40$

2.2.4. Propriedades dos Componentes dos Solos 43

2.2.5. Íons e Sais presentes nos Solos 44

2.3. Mineralogia dos Solos $\quad 49$

3 Características dos Solos Utilizados $\quad 54$

3.1. Escolha do Material Utilizado 54

3.2. Preparação dos Solos Utilizados $\quad 57$

3.3. Caracterização dos Solos Utilizados 63

3.3.1. Caracterização Mineralógica 63

3.3.2. Caracterização Química 65

3.3.3. Caracterização Física 68

3.4. Classificação dos Solos Utilizados $\quad 80$

4 Ensaios de Laboratório $\quad 82$

4.1. Preparação dos Corpos de Prova 82

4.1.1. Ensaios de Adensamento Convencional 82

4.1.2. Compactação Estática $\quad 86$

4.1.3. Compactação por Vibração 90 
4.2. Ensaios de Laboratório $\quad 91$

4.2.1. Condutividade Térmica 91

4.2.1.1. Metodologia de Ensaio 92

4.2.2. Calor Específico $\quad 94$

4.2.2.1. Metodologia de Ensaio 95

5 Apresentação e Análise dos Resultados 96

5.1. Condutividade Térmica 96

5.2. Calor Específico 123

5.3. Comparação dos Resultados Apresentados por diferentes Mineralogias 149

6 Conclusões 153

6.1. Condutividade Térmica 153

6.2. Calor Específico 155

6.3. Sugestões para Trabalhos Futuros 157

$\begin{array}{ll}\text { Referências Bibliográficas } & 158\end{array}$ 


\section{Lista de figuras}

Figura 1 - Esquema do fluxo de calor através de um elemento prismático de solo. (modificado de Bertulani, 2005)

Figura 2 - Comparações entre relações de condutividade térmica $\times$ grau de saturação (Duarte, 2004)

Figura 3 - Variação da temperatura da fonte de calor linear através do tempo 31 Figura 4 - Difusividade x Umidade Gravimétrica de argila e areia (Abu-Hamdeh, 2003).

Figura 5 - Condutividade térmica x fração de volume de ar (Tang e Cui, 2006) 48

Figura 6 - Estrutura Cristalina de argilominerais (Bastos, 2005)

Figura 7 - Estrutura das camadas e estrutura molecular dos argilominerais (Bastos, 2005) 52

Figura 8 - Localização da cidade de Prado no Sul do Estado da Bahia 55 Figura 9 - Mina de Caliman da Mineradora Monte Pascoal, Prado-BA (Bertolino, 2000)

Figura 10 - Localização das jazidas de Bentonita em Rio Negro na Argentina (Herrmann, 2001)

Figura 11 - Análise granulométrica inicial do material granular (areias quartzosas).

$\begin{array}{ll}\text { Figura } 12 \text { - Material granular } & 60\end{array}$

Figura 13 - Curvas granulométricas dos novos materiais granulares 61

Figura 14 - Difratogramas de Raios X do Caulim e da Bentonita (lâminas normal e glicolada)

Figura 15 - Visão esquemática da Capacidade de troca catiônica (Lopes e Guidolin, 1989)

Figura 16 - Procedimento complementar para ensaio de densidade dos grãos 69

Figura 17 - Resultados das sedimentações com a Bentonita utilizando procedimentos diferentes

Figura 18 - Resultados das sedimentações realizadas com C100, C80, C60 e C40

Figura 19 - Resultados das sedimentações realizadas com os solos CB80, CB60 e CB40

Figura 20 - Curvas Granulométricas dos solos AMG (real), ABG (teórica) e AU 76

Figura 21 - Resultados do ensaio de CTC pelo método de Azul de Metileno: 
Caulim (a); Bentonita (b)

Figura 22 - Prensa de Adensamento Convencional (Prensa Oedométrica) modelo Bishop

Figura 23 - Moldagem de corpo de prova para ensaio de adensamento convencional a partir de amostra deformada

Figura 24 - Curvas resultantes da etapa de carregamento dos ensaios de adensamento convencional $\quad 85$

Figura 25 - Moldes utilizados na compactação dos corpos de prova 87

Figura 26 - Moldagem de corpo de prova para compactação estática $\quad 89$

Figura 27 - Corpos de prova prontos e secando ao ar 89

Figura 28 - Molde de compactação (a) e mesa vibratória para a compactação das areias (b) 90

Figura 29 - Sonda térmica utilizada: modelo KD2-S 92

Figura 30 - Medição de parâmetros térmicos 93

Figura 31 - Curvas de condutividade térmica $x$ índice de vazios - AMG e AU

$\begin{array}{ll}\text { secas } & 97\end{array}$

Figura 32 - Curvas de condutividade térmica $x$ índice de vazios (a), índice de vazios x grau de saturação (b) - AMG $(19 \%<S<40 \%)$ e AU $(27 \%<S<45 \%)$ não saturadas

100

Figura 33 - Curva de condutividade térmica x grau de saturação - AMG $(19 \%<\mathrm{S}<40 \%)$ e AU $(27 \%<\mathrm{S}<45 \%)$ não saturadas

Figura 34 - Curvas de condutividade térmica $x$ índice de vazios (a), índice de vazios x grau de saturação (b) - AMG e AU não saturadas $(40 \%<\mathrm{S}<80 \%)$

Figura 35 - Curvas de condutividade térmica $x$ índice de vazios (a), condutividade térmica $x$ umidade gravimétrica (b) - AMG e AU saturadas

102

Figura 36 - Curva de umidade gravimétrica $x$ índice de vazios - AMG e AU saturadas

103

Figura 37 - Curvas de condutividade térmica x índice de vazios - AMG e AU em diferentes graus de saturação e umidades gravimétricas 105

Figura 38 - Curvas de condutividade térmica x umidade gravimétrica - AMG e AU para diferentes índices de vazios 106

Figura 39 - Curvas de Condutividade térmica x índice de vazios (a), umidade gravimétrica $x$ índice de vazios (b), condutividade térmica $x$ umidade gravimétrica (c) e condutividade térmica x grau de saturação (d) - C100 108 
Figura 40 - Condutividade térmica $x$ índice de vazios (a), umidade gravimétrica $x$ índice de vazios (b), condutividade térmica $x$ umidade gravimétrica (c) e condutividade térmica $x$ grau de saturação $(d)-C 80$

Figura 41 - Corpo de prova do solo CB40 após 09 dias de secagem (a) e corpo de prova do solo CB60 após 04 dias de secagem (b)

Figura 42 - Previsões da condutividade térmica da Areia Mal Graduada pelos métodos de Kersten (1949) e Johansen (1975)

Figura 43 - Previsões da condutividade térmica da Areia Uniforme pelos métodos de Kersten (1949) e Johansen (1975)

Figura 44 - Ajuste de Ewen (1988) aos dados experimentais de AMG e AU (valores médios)

Figura 45 - Comparação entre ajuste de Ewen (1988) e previsões de Kersten (1949) e Johansen (1975) para índices de vazios máximo e mínimo das areias AMG e AU

Figura 46 - Comparação entre ajuste de Ewen (1988), previsões de Kersten (1949) e Johansen (1975) com os dados experimentais do solo C100 (a) e C80 (b)

Figura 47 - Curvas de calor específico $x$ índice de vazios (a), índice de vazios $x$ grau de saturação (b) - AMG $(19 \%<S<40 \%)$ e $A U(27 \%<S<45 \%)$ não saturadas

Figura 48 - Curvas de calor específico x grau de saturação - AMG $(19 \%<\mathrm{S}<40 \%)$ e AU $(27 \%<\mathrm{S}<45 \%)$ não saturadas

Figura 49 - Curvas de calor específico $x$ índice de vazios (a), índice de vazios $x$ grau de saturação (b) - AMG e AU não saturadas (40\%<S<80\%)

Figura 50 - Curva de calor específico x grau de saturação - AMG e AU não saturadas $(40 \%<\mathrm{S}<80 \%)$

Figura 51 - Curvas de calor específico x índice de vazios (a), índice de vazios $\mathrm{x}$ umidade gravimétrica (b) - AMG e AU saturadas

Figura 52 - Curva de calor específico x umidade gravimétrica - AMG e AU saturadas

Figura 53 - Curvas de calor específico x índice de vazios - AMG e AU em diferentes graus de saturação e umidades gravimétricas

Figura 54 - Curvas de calor específico x umidade gravimétrica - AMG e AU de secas a saturadas

Figura 55 - Curvas de calor específico x umidade gravimétrica - AMG e AU para diferentes índices de vazios 
Figura 56 - Curvas de calor específico x índice de vazios (a) e calor específico x umidade gravimétrica (b) - C100 135

Figura 57 - Curva de calor específico x umidade gravimétrica - C100 135

Figura 58 - Curvas de calor específico $\mathrm{x}$ índice de vazios (a) e calor específico $\mathrm{x}$ umidade gravimétrica (b) - C80 137

Figura 59 - Curva de calor específico x umidade gravimétrica - C80 137 Figura 60 - Calor específico dos sólidos dos solos x índice de vazios - AMG e AU secas

Figura 61 - Calor específico dos sólidos $x$ índice de vazios (a), calor específico dos sólidos x grau de saturação (b) - AMG $(19 \%<S<40 \%)$ e AU $(27 \%<S<45 \%)$ não saturadas

Figura 62 - Calor específico dos sólidos $x$ índice de vazios (a), calor específico dos sólidos x grau de saturação (b) - AMG e AU não saturadas $(40 \%<\mathrm{S}<80 \%)$

Figura 63 - Calor específico dos sólidos $\mathrm{x}$ índice de vazios (a), calor específico dos sólidos x grau de saturação (b) - AMG e AU saturadas 142

Figura 64 - Difusividade Térmica x índice de vazios - AMG e AU secas 144

Figura 65 - Difusividade Térmica $x$ índice de vazios (a), difusividade térmica $x$ grau de saturação (b) - AMG $(19 \%<S<40 \%)$ e $A U(27 \%<S<45 \%)$ não saturadas

Figura 66 - Difusividade Térmica $x$ índice de vazios (a), difusividade térmica $x$ grau de saturação (b) - AMG e AU não saturadas (40\%<S<80\%)

Figura 67 - Difusividade Térmica $x$ índice de vazios (a), difusividade térmica $x$ grau de saturação (b) - AMG e AU saturadas

Figura 68 - Difusividade térmica x umidade gravimétrica - AMG e AU para diferentes índices de vazios

Figura 69 - Calor específico dos sólidos x índice de vazios (a), calor específico dos sólidos $\mathrm{x}$ umidade gravimétrica $(\mathrm{b})-\mathrm{C} 100$

Figura 70 - Calor específico dos sólidos $x$ índice de vazios (a), calor específico dos sólidos $x$ umidade gravimétrica $(b)-C 80$

Figura 71 - Difusividade Térmica $x$ índice de vazios (a), difusividade térmica $x$ umidade gravimétrica $(\mathrm{b})-\mathrm{C} 100$

Figura 72 - Difusividade Térmica $x$ índice de vazios (a), difusividade térmica $x$ umidade gravimétrica $(b)-C 80$

Figura 73 - Condutividade térmica $x$ índice de vazios para diferentes mineralogias 
Figura 74 - Calor específico x índice de vazios para diferentes mineralogias 151

Figura 75 - Comparando diferentes mineralogias em solos saturados 


\section{Lista de tabelas}

Tabela 1 - Condutividade Térmica de Solos (modificada de Hukseflux, 2005). 28

Tabela 2 - Propriedades Térmicas de Materiais distintos (modificada de Mitchell,1993) 38

Tabela 3 - Misturas de Caulim, Bentonita e Areia Quartzosa utilizadas 54

Tabela 4 - Porcentagens de areia fina, média e grossa das areias quartzosas 59

Tabela 5 - Resumo das misturas do material granular 61

Tabela 6 - Resultados da Análise Química do Caulim e da Bentonita 66

Tabela 7 - Umidade higroscópica dos Solos utilizados 68

Tabela 8 - Densidade relativa dos grãos dos solos (Gs) 69

Tabela 9 - Limites de Atterberg dos solos estudados 77

Tabela 10 - Densidades máxima e mínima dos solos AMG e AU 78

Tabela 11 - CTC e Superfície Específica do Caulim e da Bentonita. 79

Tabela 12 - Características usadas e Classificação dos solos pelo SUCS 81

Tabela 13 - Valores de Cv $\left(\mathrm{cm}^{2} / \mathrm{s}\right)$ e $\mathrm{K}(\mathrm{cm} / \mathrm{s})$ resultantes dos ensaios de adensamento $\quad 85$

Tabela 14 - Tensões de compactação aplicadas na moldagem dos corpos de prova 86

Tabela 15 - Dados dos corpos de prova e resultados de condutividade térmica $\begin{array}{ll}\text { AMG seca } & 97\end{array}$

Tabela 16 - Dados dos corpos de prova e resultados de condutividade térmica $\begin{array}{ll}\mathrm{AU} \text { seca } & 97\end{array}$

Tabela 17 - Dados dos corpos de prova e resultados de condutividade térmica AMG não saturada $\quad 99$

Tabela 18 - Dados dos corpos de prova e resultados de condutividade térmica AU não saturada

Tabela 19 - Dados dos corpos de prova e resultados de condutividade térmica AMG saturada

102

Tabela 20 - Dados dos corpos de prova e resultados de condutividade térmica AU saturada

102

Tabela 21 - Intervalos de condutividade térmica em função da variação do índice de vazios e do grau de saturação - AMG e AU

104

Tabela 22 - Variação da condutividade térmica em função da variação do teor de umidade para um mesmo índice de vazios e para índices de vazios 
diferentes - AMG

Tabela 23 - Variação da condutividade térmica em função da variação do teor de umidade para um mesmo índice de vazios e para índices de vazios diferentes - AU 106

Tabela 24 - Dados dos corpos de prova e resultados de condutividade térmica C100

108

Tabela 25 - Condutividade térmica do solo C100 na umidade higroscópica e saturado

Tabela 26 - Dados dos corpos de prova e resultados de condutividade térmica $\mathrm{C} 80$

Tabela 27 - Condutividade térmica da Bentonita na umidade higroscópica e saturada

Tabela 28 - Dados dos corpos de prova e resultados de condutividade térmica CB40 e CB60 saturados

Tabela 29 - Diferenças entre os dados experimentais de AMG e AU e as previsões de Kersten (1949) e Johansen (1975)

Tabela 30 - Ajuste de Ewen (1988) para valores médios de condutividade térmica e grau de saturação

Tabela 31 - Ajuste de Ewen para as areias Mal Graduada e Uniforme ( $e_{\text {máx }}$ e $\left.\mathrm{e}_{\text {mín }}\right)$

Tabela 32 - Diferenças entre os dados experimentais dos solos C100 e C80 e as previsões de Kersten (1949) e Johansen (1975)

Tabela 33 - Dados dos corpos de prova ensaiados e resultados de calor específico obtidos - AMG seca

Tabela 34- Dados dos corpos de prova ensaiados e resultados de calor específico obtidos - AU seca

Tabela 35 - Dados dos corpos de prova ensaiados e resultados de calor específico obtidos - AMG não saturada

Tabela 36 - Dados dos corpos de prova ensaiados e resultados de calor específico obtidos - AU não saturada

Tabela 37 - Dados de corpos de prova e resultados de calor específico - AMG saturada

Tabela 38 - Dados dos corpos de prova e resultados de calor específico - AU saturada

Tabela 39 - Intervalos de calor específico em função da variação do índice de vazios e do grau de saturação - AMG e AU 
Tabela 40 - Variação do calor específico em função da variação do teor de umidade para um mesmo índice de vazios e para índices de vazios diferentes - AMG

Tabela 41 - Variação do calor específico em função da variação do teor de umidade para um mesmo índice de vazios e para índices de vazios diferentes - AU

Tabela 42 - Dados dos corpos de prova ensaiados e resultados de calor específico obtidos - C100

Tabela 43 - Calor específico do solo C100 na umidade higroscópica e saturado

Tabela 44 - Dados dos corpos de prova ensaiados e resultados de calor específico obtidos - C80

Tabela 45 - Calor específico da Bentonita na umidade higroscópica e saturada

Tabela 46 - Dados dos corpos de prova e resultados de calor específico - CB40

e CB60 saturados

Tabela 47 - Dados dos corpos de prova e resultados de calor específico dos sólidos - AMG de seca a saturada

Tabela 48 - Dados dos corpos de prova e resultados de calor específico dos sólidos - AU de seca a saturada

Tabela 49 - Intervalos de calor específico dos sólidos em função da variação do índice de vazios e do grau de saturação - AMG e AU

Tabela 50 - Dados dos corpos de prova e resultados de calor específico dos sólidos - C100

Tabela 51 - Dados de corpos de prova e resultados de calor específico dos sólidos - C80 


\section{Lista de símbolos}

C - calor específico do solo

Ca - calor específico do ar

Cs - calor especifico dos sólidos

$C_{w}$ - calor específico da água na fase líquida

$\mathrm{C}$ - capacidade de aquecimento volumétrica do meio poroso

$\mathrm{C}_{\mathrm{a}}$ - capacidade de aquecimento volumétrico do ar

$\mathrm{C}_{s}$ - capacidade de aquecimento volumétrico dos sólidos

$\mathrm{C}_{w}$ - capacidade de aquecimento volumétrica da água na fase líquida

D - difusividade térmica

Ds - diâmetro médio dos grãos

$\Delta \mathrm{T}$ - variação de temperatura

e - índice de vazios

$\gamma$ - peso específico

$\gamma d$ - peso específico seco

$\gamma_{w}$ - peso específico da água

IP - índice de plasticidade

$\mathrm{LL}$ - limite de liquidez

LP - limite de plasticidade

$\lambda$ - condutividade térmica do solo

$\lambda q$ - condutividade térmica do quartzo

$\lambda$ sat - condutividade térmica saturada

$\lambda$ seco - condutividade térmica seca

$\lambda_{\mathrm{w}}$ - condutividade térmica da água

$\rho d$ - massa específica seca do solo

$\rho w$ - densidade da água

$S$ - grau de saturação

$\mathrm{t}$ - tempo

$\mathrm{T}$ - Temperatura

w - umidade gravimétrica 


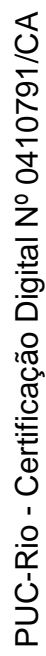

"Reaja inteligentemente mesmo a um tratamento não inteligente".

(Lao-Tsé) 